\title{
Effects of Wearing Bio-active Material Coated Fabric against $\gamma$-irradiation-induced Cellular Damage in Sprague-Dawley Rats
}

\author{
Jung Ae Kang', Hye Rim Kim', Sunhye Yoon', You Ree Nam', Sang Hyun Park ${ }^{1,2}$, Kyung-Chan Go ${ }^{3}$, Gwang-Wung Yang ${ }^{3}$, \\ Young-Hwan Rho ${ }^{3}$, Hyo-Suk Park ${ }^{3}$, Beom Su Jang ${ }^{1,2 *}$ \\ ${ }^{1}$ Korea Atomic Energy Research Institute, Jeongeup; ${ }^{2}$ Department of Radiation Biotechnology and Applied Radioisotope Science, Korea University of Science \\ and Technology, Daejeon; ${ }^{3}$ Research and Development Center, VENTEX Co. Ltd., Seoul, Korea
}

\section{Original Research}

Received August 4, 2016

Revision September 2, 2016

Accepted September 22, 2016

Corresponding author: Beom Su Jang

Division of Biotechnology, Korea Atomic Energy Research Institute, 29, Geumgu-

gil, Jeongeup 56212, Korea

Tel: +82-63-570-3372

Fax: +82-63-570-3371

E-mail:jangbs@kaeri.re.kr

This is an Open-Access article distributed under the terms of the Creative Commons Attribution NonCommercial License (http://creativecommons.org/ licenses/by-nc/4.0) which permits unrestricted noncommercial use, distribution, and reproduction in any medium, provided the original work is properly cited.

Copyright $\odot 2016$ The Korean Association for Radiation Protection

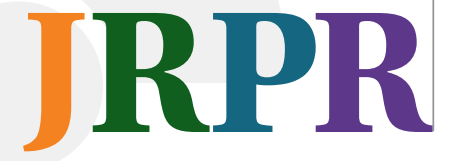

Background: Ionizing radiation causes cellular damage and death through the direct damage and/or indirectly the production of ROS, which induces oxidative stress. This study was designed to evaluate the in vivo radioprotective effects of a bio-active material coated fabric (BMCF) against $\gamma$-irradiation-induced cellular damage in Sprague-Dawley (SD) rats.

Materials and Methods: Healthy male SD rats wore bio-active material coated (concentrations in $10 \%$ and $30 \%$ ) fabric for 7 days after $3 \mathrm{~Gy}$ of $\gamma$-irradiation. Radioprotective effects were evaluated by performing various biochemical assays including spleen and thymus index, WBC count, hepatic damage marker enzymes [aspartate transaminase (AST) and alanine transaminase (ALT)] in plasma, liver antioxidant enzymes, and mitochondrial activity in muscle.

Results and Discussions: Exposure to $\gamma$-irradiation resulted in hepatocellular and immune systemic damage. Gamma-irradiation induced decreases in antioxidant enzymes. However, wearing the BMCF-30\% decreased significantly AST and ALT activities in plasma. Furthermore, wearing the BMCF-30\% increased SOD (superoxide dismutase) and mitochondrial activity.

Conclusion: These results suggest that wearing BMCF offers effective radioprotection against $\gamma$-irradiation-induced cellular damage in SD rats.

Keywords: BMCF, $\gamma$-irradiation, Cellular damage

\section{Introduction}

Humans are constantly under ionizing radiation exposures. The sources of ionizing radiation could be natural like cosmic radiation and radioactive materials like radon, or artificial including man-made radioisotope, radiotherapy, nuclear weapon test and nuclear accident. Cosmic radiation exposure levels of aircrew and passengers under environments such as high-altitude flights (above $10 \mathrm{~km}$ ), are much higher than those of medical and industrial occupations [1]. Since the March 2011 radiation accident at the Fukushima Daiichi nuclear power station (FDNPS) in Japan, which released a large amount of artificial radionuclides including ${ }^{131} \mathrm{I},{ }^{137} \mathrm{Cs}$, and ${ }^{134} \mathrm{Cs}$ [2], people are increasingly concerned about radiation causing genetic defects and, accordingly, interested in radiation protection for individual members of the public. In Korea, with increasing anxiety about radiation exposure, the 'Act on Safety Control of Radioactive Rays around 
Living Environment' has been enforced to prevent unnecessary radiation exposure in daily life [3].

The exposure ionizing radiation to the living cells can damage DNA directly or indirectly through the radiolysis of water, thereby generating reactive oxygen species (ROS) and free radicals that may damage nucleic acids, proteins, and lipids, ultimately leading to cell death or cancer [4]. The development of radioprotective clothing (e.g., made of lead, tungsten) is important for shielding patients from unnecessary exposure to radiation during radiotherapy and radiography as well as protecting the public from the effects of unintended high dose irradiation. However, radioprotective apparel is very heavy, uncomfortable, and environmentally unfriendly due to the materials used [5]. Recently, functionalized bio-active textile products were shown an improve psychological state and health of users [6]. These bio-active material coated fabrics (BMCF) are coated with 30 kinds of minerals. Clothing made of this fabric have a layer of bio-active energy, which reacts with far infrared rays and activates muscle movement by transferring the energy to the muscle [7]. There are no reports available on the efficacy of BMCF against $\gamma$-irradiation-induced cellular damage through in vivo studies. In the present study, we investigated the protective effects of wearing such material coated fabric against $\gamma$-irradiation-induced cellular damage in SD rats.

\section{Materials and Methods}

\section{BMCF}

The fabric samples were provided by VENTEX (Seoul, Korea). The bio-active material contained 30 kinds of minerals, such as $\mathrm{SiO}_{2}, \mathrm{Mg}, \mathrm{Al}_{2} \mathrm{O}_{3}, \mathrm{Na}, \mathrm{Ca}$, and $\mathrm{Fe}_{2} \mathrm{O}_{3}$.

\section{Animals}

Six-week-old male SD rats were purchased from Orient Bio Inc., Seoul, Korea. All animals were acclimatized to controlled conditions of temperature $\left(23 \pm 2^{\circ} \mathrm{C}\right)$, humidity $(55 \pm 5$ $\%)$, and light (12 $\mathrm{h}$ light/dark cycle) at the RI-Biomics animal research laboratory, Advanced Radiation Technology Institute (ARTI), Korea Atomic Energy Institute (KAERI). The animals were provided with a sterile pellet diet and water ad libitum. The study was approved by the Institutional Animal Ethical Committee and performed in strict compliance with the guidelines prescribed by the committee (KAERI-IACUC-2015-002).

\section{Experimental design and irradiation}

The animals were divided into four groups with five animals in each group. Unanesthetized animals were placed in a well-ventilated acrylic restrainer and exposed to a dose of 3 Gy whole-body $\gamma$-radiation at a dose rate of $1.1 \mathrm{~Gy} \cdot \mathrm{min}^{-1}$ using a Gammacell 40 Exactor (MDS Nordion, Ottawa, Canada) at ARTI, KAERI. Immediately after irradiation, the animals were fitted with fabric vests. The normal and irradiation groups wore conventional fabric vests, and the experimental groups wore concentrations of BMCF- $10 \%$ and $-30 \%$ in fabric vests for 7 consecutive days.

The experimental design and treatment protocol were as follows:

Normal group: The rats were fitted with conventional fabric vests for 7 days without $\gamma$-irradiation.

Irradiation group: The rats were exposed to $3 \mathrm{~Gy}$ of $\gamma$-irradiation and fitted with conventional fabric vests for 7 days.

Irradiation+BMCF-10\%: Rats were exposed to $3 \mathrm{~Gy}$ of $\gamma$-irradiation and fitted with BMCF-10\% fabric vests for 7 days.

Irradiation+BMCF-30\%: Rats were exposed to $3 \mathrm{~Gy}$ of $\gamma$-irradiation and fitted with BMCF-30\% fabric vests for 7 days.

\section{Blood and tissue collection}

Twenty-four hours post irradiation, overnight-fasted animals were anesthetized with isoflurane and blood was collected from the inferior vena cava using a syringe. The animals were then euthanized by exsanguination, and the liver, spleen, and thymus were excised and weighted.

The spleen and thymus indexes were calculated as follows:

Spleen index $=($ spleen weight $/$ body weight $) \times 100$

Thymus index $=($ thymus weight $/$ body weight $) \times 100$

\section{Determination of WBC, RBC, platelet, AST, and ALT concentrations}

White blood cells (WBC), red blood cells (RBC) and platelets were automatically counted using a hematology analyzer (HEMAVET HV950; Drew Scientific, Inc., Dallas, TX). The blood was collected in heparinized tubes, and plasma was separated by centrifugation (1,000 $\mathrm{g}$ for 20 minutes) and stored at $4^{\circ} \mathrm{C}$. Aspartate and alanine transaminases (AST and ALT) in plasma were assayed using enzymatic kits (Asan Pharmaceuticals, Co., Ltd., Seoul, Korea). 


\section{Evaluation of oxidative stress and antioxidant defense system}

Liver tissue was homogenized in a phosphate buffer $(0.1$ M; pH 7.4) using a Precellys 24-dual homogenizer (Bertin, Villeurbanne, France). Superoxide dismutase (SOD), catalase, and glutathione peroxidase (GPx) in liver tissue were assayed using enzymatic assay kits (Biovision, Inc., Milpitas, CA) as the manufacturer's instructions.

\section{Mitochondrial activity}

Subtrapezial muscle tissue was rapidly removed and homogenized in a phosphate buffer (0.1 M; pH 7.4) using a Precellys 24-dual homogenizer. Mitochondrial activity was assayed using a recently developed sensitive spectrophotometric method [8]. In brief, $50 \mu \mathrm{g}(20 \mu \mathrm{L})$ of mitochondrial proteins were suspended in $960 \mu \mathrm{L}$ of medium containing 20 $\mathrm{mM} \mathrm{KH} \mathrm{PO}_{4}, 3.5 \mathrm{~g} \cdot \mathrm{L}^{-1} \mathrm{BSA}, 60 \mu \mathrm{M}$ 2,6-dichloroindophenol, $70 \mu \mathrm{M}$ decylubiquinone, $1 \mu \mathrm{M}$ antimycin-A at $30^{\circ} \mathrm{C}$. After 3 minutes, $20 \mu \mathrm{L}$ of $10 \mathrm{mM}$ NADH was added, and absorbance was measured at 1 minute intervals for 4 minutes at $37^{\circ} \mathrm{C}$. After that, $1 \mu \mathrm{L}$ rotenone ( $1 \mathrm{mM}$ in DMSO) was immediately added, and the absorbance was measured again at 1 minute intervals for an additional 4 minutes.

\section{Statistical analysis}

Statistical analyses were conducted using a one-way analysis of variance (ANOVA), and inter group comparisons were made using Tukey's multiple comparison test. The values are expressed as the mean \pm S.D. of five samples per group. A $P<0.05$ was considered significant.

\section{Results and Discussion}

1. Effects of wearing BMCF on body weight, spleen, and thymus indexes

The immune system is very sensitive to ionizing radiation. Radiation may interrupt the immune system, resulting in organism infection, bleeding, and various diseases [9]. Table 1 shows the effect of wearing BMCF on body weight, spleen, and thymus indexes in rats exposed to 3 Gy $\gamma$-irradiation. Gamma-irradiated rats showed a significant $(p<0.05)$ decrease in body weight, spleen, and thymus indexes compared with those of normal rats. Wearing BMCF-30\% for 7 days significantly $(p<0.05)$ improved their body weight, spleen and thymus indexes compared with those of $\gamma$ irradiated rats. These results showed that wearing BMCF$30 \%$ significantly improved the spleen and thymus indexes, compared with those of irradiated rats, indicating a protective effect on the immune system.

\section{Effects of wearing BMCF on hematology}

Table 2 shows the effect of wearing BMCF on WBC, RBC, and platelets in rats exposed to $3 \mathrm{~Gy} \gamma$-irradiation. Gammairradiated rats showed a significant $(p<0.05)$ decrease in WBC, RBC, and platelet count compared with those of normal rats. Wearing BMCF-30\% for 7 days significantly $(p<$

Table 1. Effects of Wearing BMCF on the Status of Body Weight, Spleen, and Thymus Indexes in SD Rats Exposed to 3 Gy of Irradiation

\begin{tabular}{lll}
\hline Groups & Body weight $(\mathrm{g})$ & Spleen index \\
\hline Normal & $189.90 \pm 11.44$ & $0.204 \pm 0.012$ \\
Irradiation & $172.93 \pm 7.57^{\#}$ & $0.096 \pm 0.006^{\# \#}$ \\
Irradiation+BMCF-10\% & $185.45 \pm 6.50$ & $0.108 \pm 0.009$ \\
Irradiation+BMCF-30\% & $189.25 \pm 6.42^{*}$ & $0.078 \pm 0.010^{\# \#}$ \\
\hline
\end{tabular}

Values are expressed as the mean \pm S.D. for five rats in each group.

$\# p<0.05$ and ${ }^{\#} p<0.01$ as compared with normal and 3 Gy of irradiation group.

${ }^{*} p<0.05$ and ${ }^{* *} p<0.01$ represent significant differences compared with 3 Gy of irradiation group.

Table 2. Effects of Wearing BMCF on the Status of White Blood Cell, Red Blood Cell, and Platelet in SD Rats Exposed to 3 Gy of Irradiation

\begin{tabular}{|c|c|c|c|}
\hline Groups & White blood cell $\left(\times 10^{3}\right.$ cells $\left.\cdot \mu \mathrm{L}^{-1}\right)$ & Red blood cell $\left(\times 10^{3}\right.$ cells $\left.\cdot \mu L^{-1}\right)$ & Platelet $\left(\times 10^{3}\right.$ cells $\left.\cdot \mu L^{-1}\right)$ \\
\hline Normal & $5.87 \pm 1.29$ & $8.38 \pm 0.27$ & $1366.50 \pm 39.50$ \\
\hline Irradiation & $1.41 \pm 0.51^{\# \#}$ & $7.89 \pm 0.25 \#$ & $749.75 \pm 132.07^{\# \#}$ \\
\hline Irradiation+BMCF-10\% & $1.28 \pm 0.09$ & $8.16 \pm 0.48$ & $945.50 \pm 52.60$ \\
\hline Irradiation+BMCF-30\% & $1.85 \pm 0.28$ & $7.05 \pm 1.73$ & $987.00 \pm 176.10^{\star \star}$ \\
\hline
\end{tabular}

Values are expressed as the mean \pm S.D. for five rats in each group.

$\# p<0.05$ and ${ }^{\# \#} p<0.01$ as compared with normal and 3 Gy of irradiation group.

${ }^{*} p<0.05$ and ${ }^{* \star} p<0.01$ represent significant differences compared with 3 Gy of irradiation group. 
0.05) improved their platelets of test rats compared with those of irradiated rats.

\section{Effects of wearing BMCF on hepatocellular damage}

In the present investigation, exposure of rats to $3 \mathrm{~Gy}$ of $\gamma$-irradiation significantly $(p<0.05)$ increased the activity of ALT and AST in plasma compared with those observed in normal rats. This might be attributed to the rapid release of these enzymes from the cytoplasm into blood circulation after rupture of plasma membranes and cellular leakage [10]. The effects of wearing BMCF on the status of hepatic marker enzymes in plasma are presented in Figure 1. Wearing BMCF-30\% significantly $(p<0.05)$ attenuated these increases in ALT and AST in plasma compared with those of irradiated rats and appeared to prevent liver damage.

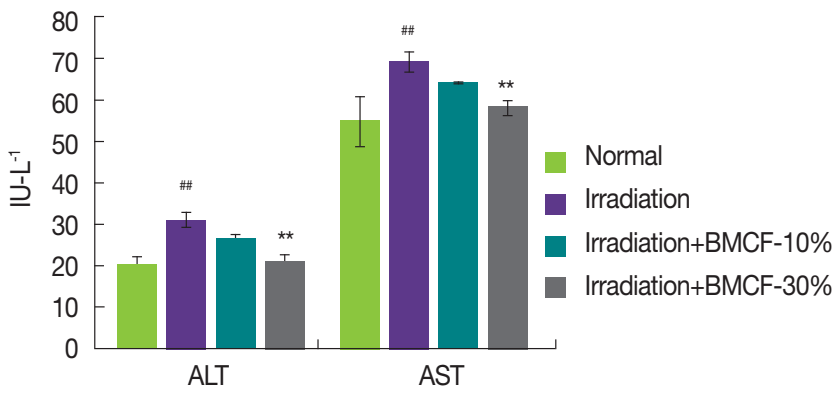

Fig. 1. Effects of wearing BMCF on the status of ALT and AST in plasma of normal, irradiated, and wearing BMCF-10 and -30\% irradiated SD rats. Values are expressed as the mean \pm S.D. for five rats in each group.

${ }^{\#} p<0.05$ and ${ }^{\# \#} p<0.01$ as compared with normal and 3 Gy of irradiation group.

${ }^{*} p<0.05$ and ${ }^{* *} p<0.01$ represent significant differences compared with 3 Gy of irradiation group.

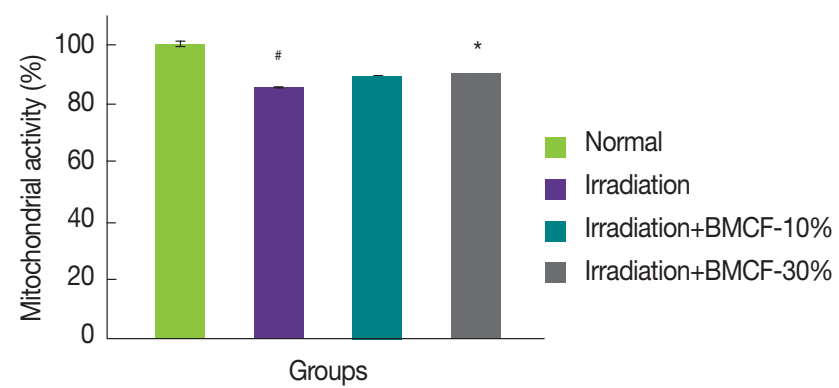

Fig. 3. Effects of wearing BMCF on the status of mitochondrial activity in the muscles of the back of normal, irradiated, and wearing BMCF-10 and $-30 \%$ irradiated SD rats.

Values are expressed as the mean \pm S.D. for five rats in each group. ${ }^{\#} p<0.05$ and ${ }^{\# \#} p<0.01$ as compared with normal and 3 Gy of irradiation group.

${ }^{*} p<0.05$ and ${ }^{* *} p<0.01$ represent significant differences compared with 3 Gy of irradiation group.

\section{Effects of wearing BMCF on antioxidant defense system in liver tissue}

Radiation-induced ROS affect the antioxidant defense mechanism by reducing the intracellular concentration of SOD, catalase, and GPx [11]. Figure 2 shows the changes in the levels of SOD, catalase, and GPx in the liver tissue of normal, irradiated, and wearing BMCF-10 and -30\% irradiated rats. The activities of SOD, catalase, and GPx significantly $(p<0.05)$ decreased in rats exposed to 3 Gy of $\gamma$-irradiation compared with those of normal rats. Wearing BMCF-30\%
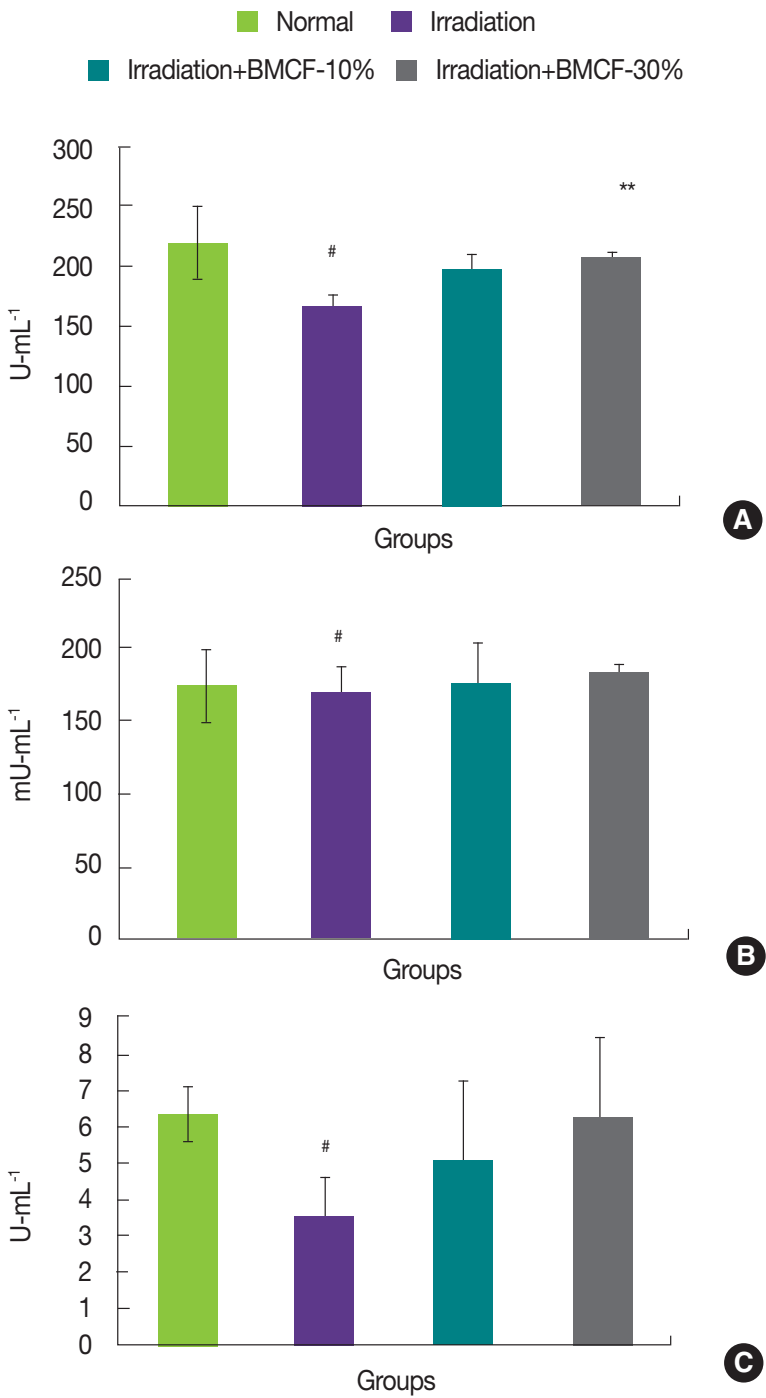

C

Fig. 2. Effects of wearing BMCF on the status of SOD (A), catalase (B), and GPx (C) in the liver tissue of normal, irradiated, and wearing BMCF-10 and $-30 \%$ irradiated SD rats. Values are expressed as the mean \pm S.D. for five rats in each group.

${ }^{\#} p<0.05$ and ${ }^{\# \#} p<0.01$ as compared with normal and 3 Gy of irradiation group.

${ }^{*} p<0.05$ and ${ }^{* *} p<0.01$ represent significant differences compared with 3 Gy of irradiation group. 
significantly $(p<0.05)$ increased SOD compared with those of irradiated rats. Thus, BMCF contributes to the intracellular antioxidant defense system.

\section{Effects of wearing BMCF on mitochondrial activity in muscle}

ROS induced by ionizing radiation can destroy mitochondria and thereby lead to apoptosis [12]. Figure 3 shows the changes in mitochondrial activity levels in the muscle of normal, irradiated, and wearing BMCF- 10 and 30\% irradiated rats. Mitochondrial activity decreased significantly $(p<0.05)$ in rats exposed to 3 Gy of $\gamma$-irradiation compared with those of normal rats. Wearing BMCF-30\% significantly $(p<0.05)$ increased mitochondrial activity compared with those of irradiated rats.

The protective clothing used in a variety of applications including radiotherapy and radiography was made of lead and tungsten. Since there were often objections to the heaviness and uncomfortable of the radioprotection apparel, alternative shielding materials that are lighter have been sought [13, 14]. These bio-active material coated fabrics (BMCF) are made lighter and more breathable, the irradiation-induced cellular damage is significantly decreased.

\section{Conclusion}

In this study, we demonstrated the protective effects of bio-active material coated fabric against $\gamma$-irradiationinduced cellular damage in SD rats. It was observed that BMCF vests prevented the development of irradiation-induced oxidative stresses. It is anticipated that BMCF would be extensively use in the manufacture of new generation radiprotective clothes.

\section{Acknowledgement}

This work was supported by a Nuclear Research Development Program of the National Research Foundation (2012 M2A2A6011335) grant funded by the Korea Ministry of Science ICT Future planning.

\section{References}

1. Dyer CS, Lei F, Clucas SN, Smart DF, Shea MA. Solar particle enhancements of single event effect rates at aircraft altitude. IEEE Tran. Nucl. Sci. 2003;50:2038-2045.

2. Kato H, Onda Y, Teramage M. Depth distribution of ${ }^{137} \mathrm{Cs}$, ${ }^{134} \mathrm{Cs}$, and ${ }^{131} \mathrm{I}$ in soil profile after Fujushima Daiichi Nuclear Power Plant Accident. J. Environ. Radioact. 2011;111:59-64.

3. Jeong C, Oh H, Lee J, Jo S, Park S. Analysis of dose by items according to act on safety control of radiation around living environment. J. Korea Soc. Radiol. 2013;7(6):377-381.

4. Hall EJ, Giaccia AJ. Radiobiology for the radiologist. 6th Ed. New York NY. Lippincott Williams and Wilkins. 2006;5-15.

5. Fan WC, Drumm CR, Roeske SB, Scrivner GJ. Shielding considerations for satellite microelectronics. IEEE Trans. Nucl. Sci. 1996;43(6):2790-2796.

6. Lee MS, Kim HJ, Song J, Park KW, Moon SR. Effects of multifunctional fabric on cardiac autonomic tone and psychological state. Int. J. Neurosci. 2004;114:923-931.

7. Lee DH, et al. Improvement characteristics of bio-active materials coated fabric on rat muscular mitochondria. Korean J. Physiol. Pharmcol. 2015;19:283-289.

8. Janssen AJ, Trijbels FJ, Sengers RC, Smeitink JA, van den Heuvel LP, Winties LT, Stoltenborg-Hogenkamp BJ, Rodenburg RJ. Spectrophotometric assay for complex I of the respiratory chain in tissue samples and cultured fibroblasts. Clin. Chem. 2007;53: 729-734.

9. Zhao H, Wang Z, Ma F, Yang Xin, Cheng C, Yao L. Protective effect of anthocyanin from Lonicera Caerulea var. edulis on radiation-induced damage in mice. Int. J. Mol. Sci. 2012;13:1177311782.

10. Sallie R, Tredger JM, Willam R. Drugs and the liver. Biopharm. Drug Dispos. 1991;12: 251-259.

11. Samarth RM, Kumar A. Radioprotection of Swiss albino mice by plant extract Menthe piperita (Linn.). J. Radiat. Res. 2003;44:101109.

12. Mikinory K, Taketoshi A, Koichi N, Osamu I. Regulation of cell survival and death signaling induced by oxidative stress. J. Clin. Biochem. Nutr. 2008;43:51-57.

13. Webster EW. Experiments with medium-Z materials for shielding against low-energy $x$ rays. Radiology. 1966;84:146.

14. Yaffe M, Mawdsley GE, Lilley M, Servant R, Reh G. Composite materials for x-ray protection. Health Phys. 1991;60(5):661-664. 